\title{
43 Obesity and mortality risk
}

According to the data from many large-scale studies, marked obesity is associated with an increased risk of mortality (Greenberg 2013). A metaanalysis of prospective studies on this subject gave special consideration to the age, sex and smoking status of 894,576 participants (Whitlock et al. 2009). The findings showed that obesity, reflected in a BMI between 30 and 35, reduced life expectancy by 2-4 years. A total of 8-10 years of life were lost when the person's weight produced a BMI between 40 and 45. The latter decreased life expectancy is approximately equal to the effect of smoking. Another large meta-analysis by the National Cancer Institutes in the USA covering 19 prospective studies with data from nearly 1.5 million adults of European origin and observation periods of 5-28 years yielded the same results (Gonzales et al. 2010).

Since BMI is not the most optimal measure for determining the amount of abdominal fat mass that can cause illness, overweight persons with fat mass distributed over the whole body or very muscular athletes even with a BMI of 25-29.9 can have a longer life expectancy than people with normal weight (Flegal et al. 2013). Conversely, when the accumulation of fat pads in the abdominal organs and especially in the liver becomes too large, the mortality rates are higher, even in normal-weight or underweight persons (Zhang et al. 2008, Cerhan et al. 2014). The results of the Framingham Heart Study (Britton et al. 2013) and the EPIC study (van der A et al. 2014) have confirmed that large amounts of abdominal fat are related to higher mortality rates.

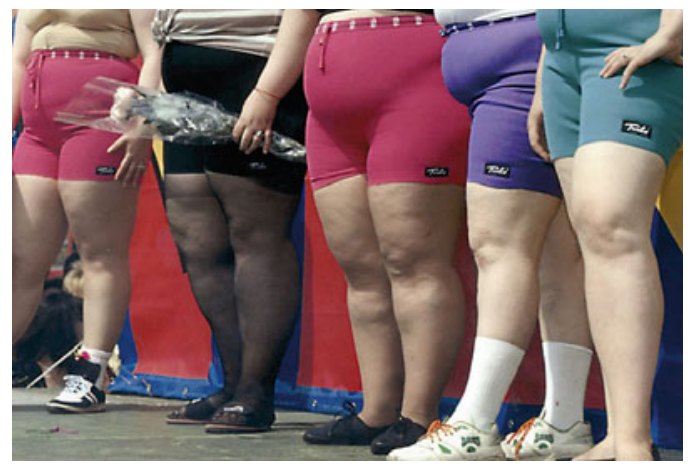

- Fig. 43.1 Source: dpa/akg

A too high proportion of abdominal fat minimized by surgical interventions can confer significant health benefits (Adams et al. 2012, O'Brien et al. 2013). This was also shown by the Swedish Obese Subjects Study, ongoing since 1981, concerning the relationship between body weight and mortality (Sjöström et al. 2007, 2012, 2014, Carlsson et al. 2012). The authors used interventions based on bariatric surgery to lower the weight of people with morbid obesity. In these patients, they performed gastric bypass surgery, which leaves only a smaller portion of the stomach remaining for nutritional uptake, or they shortened their intestine. Then, they compared this study population with a cohort of obese controls who did not undergo surgery. Only those patients who underwent surgery achieved substantial weight loss and had a markedly reduced incidence of type 2 diabetes, myocardial infarction and stroke, as well as lower overall mortality. 